\title{
Construction System of Heavy Parts by the Coordinated Control between a Crane and a Robot
}

\author{
Tamio ARAI, Hisashi OSUMI \\ Department of Precision Machinery Engineering \\ Faculty of Engineering \\ The University of Tokyo \\ 7-3-1 Hongo, Bunkyo-ku, TOKYO 113, JAPAN
}

\begin{abstract}
Automatic assembly of heavy parts for construction will be the next step of assembly automation. This paper deals with a coordinated control between a crane and an industrial robot for assembling heavy parts. The suspended part by the crane and the flexibility of the robot are modeled by stiffness matrices. The design of a flexible mechanism installed to the end-effector of the robot is studied. The closed-loop mechanism consisted of the crane and the robot is analyzed in kinematics. Since the system has redundancy in kinematics, an algorithm of inverse kinematics is proposed and verified by experiment. This robot system provides a fundamental technology in assembly of heavy parts.
\end{abstract}

\section{INTRODUCTION}

Automatic assembly of small parts can be easily achieved by using a robot. However, that of heavy parts in construction sites are still left behind. The reasons are

(1) handling devices are too large and expensive to install in a site,

(2) safety requirements are very strong.

This paper deals with an automatic handling system for heavy parts in construction by means of a coordinated control between a crane and an industrial robot as illustrated in Fig.1. The former compensates the effect of the gravity and the latter makes a heavy part move precisely as if the part were light. To realize the coordinated control between a crane and a PID position-controlled robot, it is necessary to install a flexible mechanism of heavy parts.

\section{COORDINATED CONTROL SYSTEM BETWEEN A CRANE AND A ROBOT}

Demands for heavy parts assembly can be seen in various industries, especially shipyards, heavy electric industries, and constructions. The current status of heavy part assembly, however, is to utilize just manual machines such as folk lifts, balancers and cranes

The basic specifications for heavy part s assembly are (1) large payload over 1 ton, (2) large working space over 10 meters, (3) relatively high repeatability such as $1 \mathrm{~mm}$. Additionally, (4) part feeding and (5) safety are the key issues. To satisfy the specifications(1), (2) and (4) in low cost, a wire suspending system such as a crane is indispensable. However, since it causes vibration in itself, a robot is necessary to secure the positioning accuracy and to ensure the universality in handling. So, we propose the combination of a crane and a small-size robot as one of the best solutions.

Research in this field has been rare. Matsui ${ }^{1)}$ reported an assembly system with a balancer and a robot. Patarinski ${ }^{2}$ ) solved kinematics. Authors made vibration control of the combination of a crane and a robot 3$), 4$ ).

\section{DESIGN OF COORDINATED SYSTEM BETWEEN A CRANE AND A ROBOT}

In order to realize a coordinated control system between two positioning devices, compliance is evidently necessary to avoid excessive internal force generated by

[Static error] mutual positioning errors statically, and

[Dynamic error] vibrations at the positioning dynamically.

To implement compliance, either of accommodation methods

[Active] using servo control to make the low servo stiffness,

[Passive] installing a mechanism with low mechanical stiffness, 

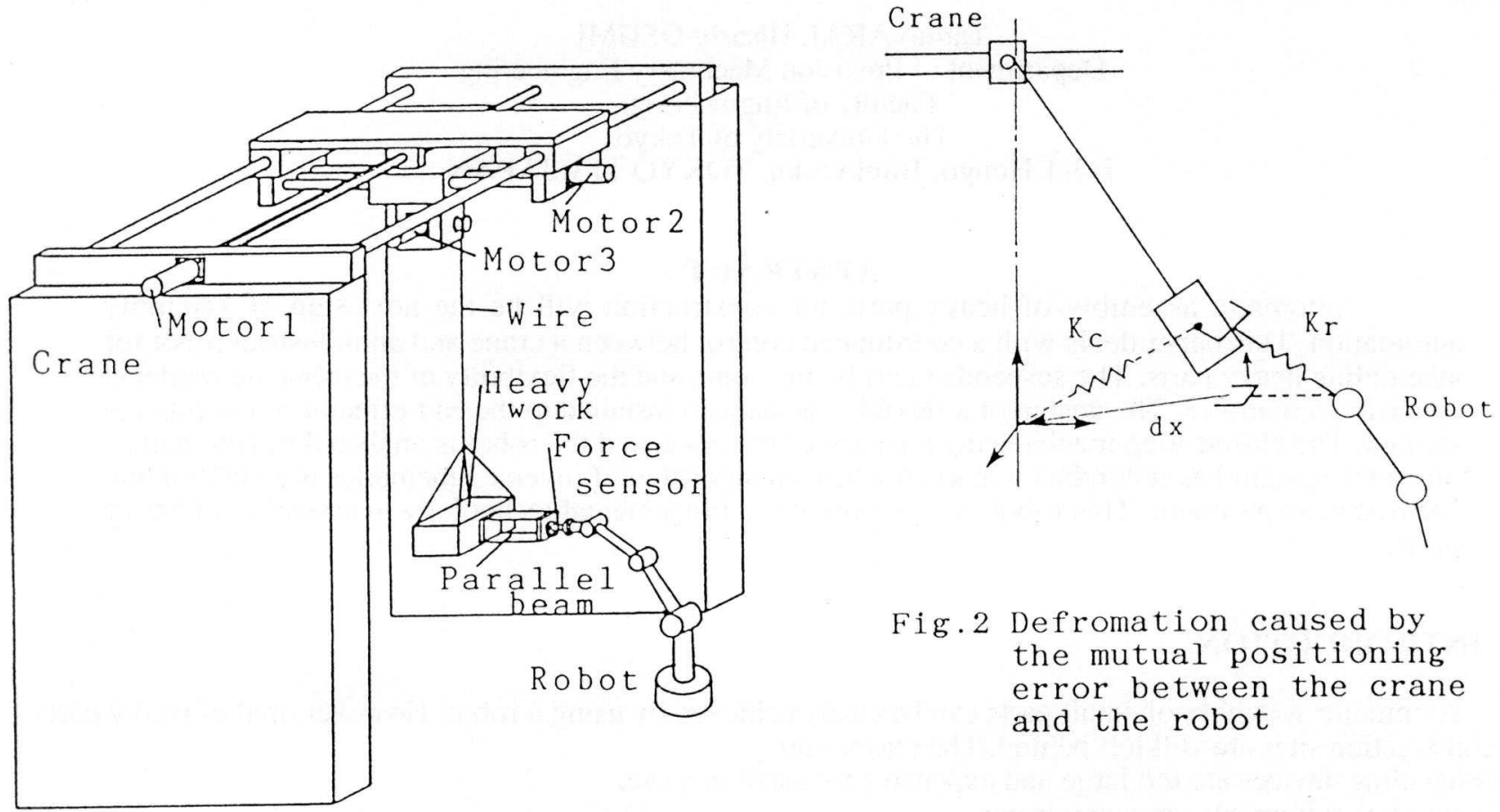

Fig.2 Defromation caused by the mutual positioning error between the crane and the robot

Fig.1 The coordinated system between a crane and a robot

will be introduced. For conventional industrial robots, however, the latter is more practical than the former because of the slow response and large friction forces at joints and gears.

Let us discuss the design of mechanical flexibility. The suspended part is modeled as a part supported by springs, whose stiffness is represented by the stiffness matrix, $\mathrm{K}_{\mathrm{C}}$. Let us consider what compliance is required on the robot. Let $\mathrm{K}_{\mathrm{r}}$ denote the stiffness matrix of the robot side and $\mathrm{dx}$ denote the mutual positioning error as shown in Fig.2. Then, the minimum static requirement is

$\mathrm{K}_{\mathrm{r}}\left(\mathrm{K}_{\mathrm{c}}+\mathrm{K}_{\mathrm{r}}\right)^{-1} \mathrm{~K}_{\mathrm{c}} \mathrm{dx} \leq \mathrm{F}_{\mathrm{r}}$

where $\mathbf{F}_{\mathbf{r}}$ is the capability of the force by the robot at the end-effector. $\mathrm{K}_{\mathrm{r}}$ must be designed to satisfy this inequality. We assumed that the strength of the robot is much larger than $\mathrm{K}_{\mathrm{r}}$. Generally speaking, dynamic requirement for the accommodation to vibration is larger than the static requirement, thus $\mathrm{K}_{\mathrm{r}}$ should be designed with consideration of response time of feedback system

So, the following schemes are possible in the implementation of the compliance installed at the tip of the end-effector.

[Huge mass system] In the case of a huge mass against a robot: As the motion of the mass is apt to destroy the end-effector, the compliance is required in all degrees of freedom as illustrated in Fig.3. This idea has been discussed in Author's previous research 3$), 4$ ).

[Large mass system] In the case of a large mass against a robot: The robot itself can control the vibration. Thus, additional compliance is required only to coordinate the static positioning error. In this research, we put only one flexible mechanism in the direction of the wire at the tip of end-effector as illustrated in Fig.1. 


\subsection{Modeling of a Crane}

Since the suspension system is a pendulum, it can be modeled as a stiffness matrix $\mathrm{K}_{\mathrm{C}}$ defined in the coordinate system $\Sigma_{c}$ as illustrated in Fig.4.

$\mathrm{K}_{\mathrm{c}}=\left[\begin{array}{cccccc}\mathrm{k}_{\mathrm{x}} & 0 & 0 & 0 & \mathrm{k}_{\mathrm{xb}} & 0 \\ 0 & \mathrm{k}_{\mathrm{y}} & 0 & \mathrm{k}_{\mathrm{ya}} & 0 & 0 \\ 0 & 0 & \mathrm{k}_{\mathrm{z}} & 0 & 0 & 0 \\ 0 & \mathrm{k}_{\mathrm{ya}} & 0 & \mathrm{k}_{\mathrm{a}} & 0 & 0 \\ \mathrm{k}_{\mathrm{xb}} & 0 & 0 & 0 & \mathrm{k}_{\mathrm{b}} & 0 \\ 0 & 0 & 0 & 0 & 0 & \mathrm{k}_{\mathrm{c}}\end{array}\right]$

$\mathrm{k}_{\mathrm{x}}=\mathrm{k}_{\mathrm{y}}=\frac{\mathrm{mg}}{\mathrm{L}}$

$\mathrm{k}_{\mathrm{xb}}=-\mathrm{k}_{\mathrm{ya}}=\frac{\mathrm{dmg}}{\mathrm{L}}$

$\mathrm{k}_{\mathrm{a}}=\mathrm{k}_{\mathrm{b}}=\frac{\mathrm{d}(\mathrm{L}+\mathrm{d}) \mathrm{mg}}{\mathrm{L}}$

where $k_{Z}$ is the tensile stiffness of the wire, $k_{C}$ is the torsional stiffness about the wire, $m$ is the mass of the part, and $\mathrm{g}$ is the acceleration of gravity. Each component except $\mathrm{k}_{\mathrm{C}}$ and $\mathrm{k}_{\mathrm{Z}}$ is proportional to the mass of the suspended part. As discussed in previous section, the necessity of compliance on the robot side is judged according to the size and the direction of large stiffness of the pendulum.

A stiffness matrix $K_{C}$ can be converted into a diagonal one by means of congruence transformation with an orthogonal matrix U:

$$
U^{\mathrm{T}} \mathrm{K}_{\mathrm{c}} \mathrm{U}=\operatorname{diag}\left(\mathrm{I}_{1} \mathrm{l}_{2} \mathrm{I}_{3} \mathrm{l}_{4} \mathrm{l}_{5} \mathrm{l}_{6}\right)
$$

When the mass $m$ is very large, all axes except the torsional axis have high stiffness and thus flexible mechanism in multiple axes must be introduced on the robot side as illustrated in Fig.3. On the contrary, when the mass is not very large, 5 axes except for the tensile direction of the wire can be assumed to have flexibility on the robot side. Therefore, only the vertical direction (the $\mathrm{z}$ axis) requires the flexibility. After this, the system is defined to have the mechanism illustrated in Fig.1.

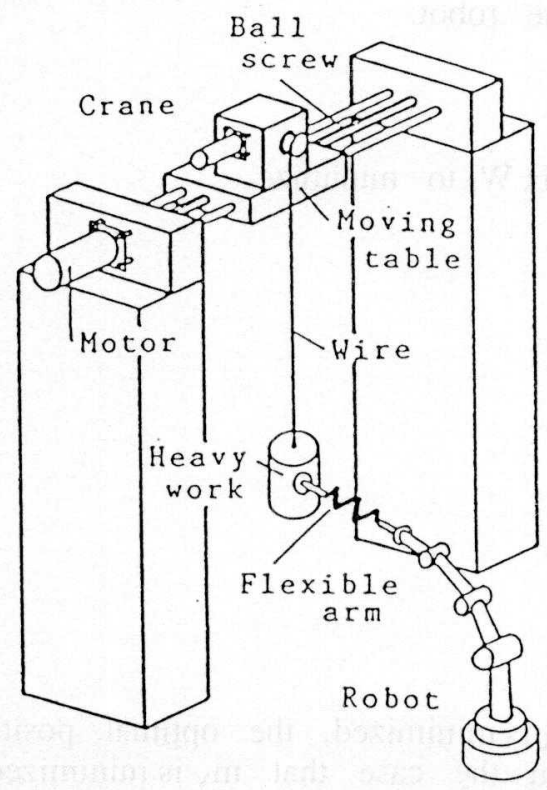

Fig. 3 Heavy parts assembly system for a huge mass

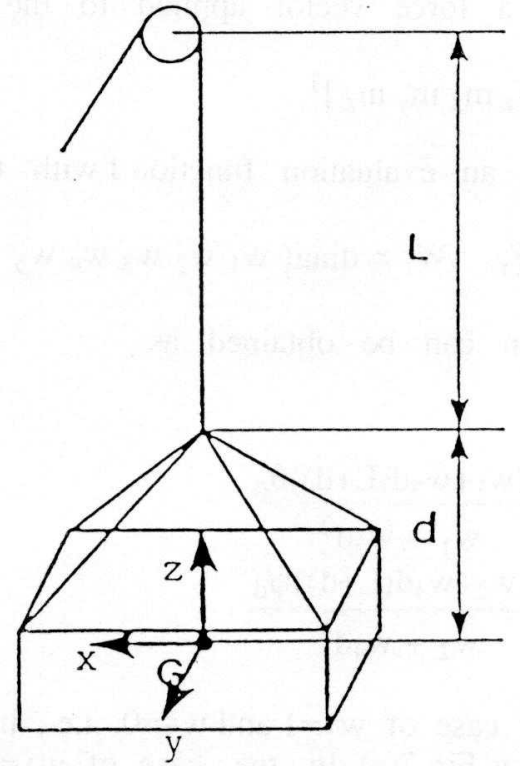

Fig.4 Parameters and the coordinate system on the suspended part 


\subsection{The Kinematic Analysis of a Mechanism with Flexibility}

When a robot grasps the suspended part by a crane, these mechanisms produce a closed loop totally. The number of the allowed degrees of freedom, $n$, is defined as

$$
n=6(p+r-1)-5(p+r)=(p+r)-6
$$

where $p$ and $r$ are the numbers of links in the crane and the robot. When a mechanism contains flexibility, it is modeled as virtual links connected with virtual joints; a prismatic joint is assumed for translational flexibility and a rotational joint for rotational one as illustrated in Fig.5. Assume that the crane and the robot may have $\mathrm{p} 1$ and $\mathrm{r}_{1}$ respectively as virtual degrees of freedom, and that the cross section of the two elasticity spaces defined by the eigenvectors in 3.1 may have $h$ degrees of freedom. Then, Eq.(4) is rewritten as

$n=6\left(p+r+p_{1}+r_{1}-1\right)-5\left(p+r+p_{1}+r_{1}\right)-h=\left(p+r+p_{1}+r_{1}\right)-6-h$

Let us check the coordinated system in this study. The robot has $r=6$ on joints and $r 1=1$ for flexibility at the end-effector. The crane has $\mathrm{p}=3$ and $\mathrm{pl}_{\mathrm{l}}=5$ as illustrated in Fig.6. $\mathrm{h}$ is 0 in this mechanism. Then $\mathrm{n}$ becomes 9 . Therefore, even though the pose of the part, i.e., 6 degrees of freedom, is given definitely, 3 degrees of freedom are still left as redundant degrees of freedom. To solve the inverse kinematics from the pose of the part to the joint angles in this mechanism, an algorithm with an evaluation function is required.

\subsection{Inverse Kinematics of the Coordinated System}

We propose an algorithm to determine the configuration values of both the crane and the robot by means of minimizing the square sum of the forces and the moments applied to the end-effector of the robot.

Now we choose the representing 3 parameters for redundant degrees of freedom the displacements of the crane, $x_{c}$ and $y_{c}$, and the displacement of the parallel beam, qr. Let $x d$ denote the coordinate of the desired pose of the suspended part, assuming the orientation angles to be infinitesimal,

$x_{d}=\left[x_{d} y_{d} z_{d} \psi_{d} \theta_{d} \phi_{d}\right]^{T}$

Let $\mathbf{F}_{\mathrm{r}}$ be a force vector applied to the end-effector of the robot.

$\mathbf{F}_{\mathrm{r}}=\left[\mathrm{f}_{\mathrm{x}} \mathrm{f}_{\mathrm{y}} \mathrm{f}_{\mathrm{z}} \mathrm{m}_{\mathrm{x}} \mathrm{m}_{\mathrm{y}} \mathrm{m}_{\mathrm{z}}\right]^{\mathrm{T}}$

Introducing an evaluation function $\mathrm{J}$ with the weighting matrix $\mathrm{W}_{\mathrm{r}}$ to minimize

$\mathrm{J}=\mathbf{F}_{\mathrm{r}}^{\mathrm{T}} \mathrm{W}_{\mathrm{r}} \mathbf{F}_{\mathrm{r}}, \quad \mathrm{W}_{\mathrm{r}}=\operatorname{diag}\left(\mathrm{w}_{1} \mathrm{w}_{2} \mathrm{w}_{3} \mathrm{~W}_{4} \mathrm{w}_{5} \mathrm{w}_{6}\right)$

the solution can be obtained as

$\mathrm{q}_{\mathrm{r}}=0$

$x_{c}=x_{d}+\frac{d\left(w_{1}+w_{5} d(L+d)\right) \theta_{d}}{w_{1}+w_{5} d^{2}}$

$y_{c}=y_{d}-\frac{d\left(w_{2}+w_{4} d(L+d)\right) \psi_{d}}{w_{2}+w_{4} d^{2}}$

In the case of $w_{1}=1$ and $w_{5}=0$, i.e., in the case that $f_{X}$ is minimized, the optimal position is illustrated as Fig.7(a). In the case of $\mathrm{w}_{1}=0$ and $\mathrm{w}_{5}=1$, i.e., in the case that $\mathrm{m}_{\mathrm{y}}$ is minimized, the position is illustrated as Fig.7(b). In the same manner, the optimal position about the $y$ axis is determined. In order to have a general and reasonable set of weighting of the evaluation function, the idea of manipulating force ellipsoid of the robot $^{5)}$ may be one of the most appropriate candidates. 


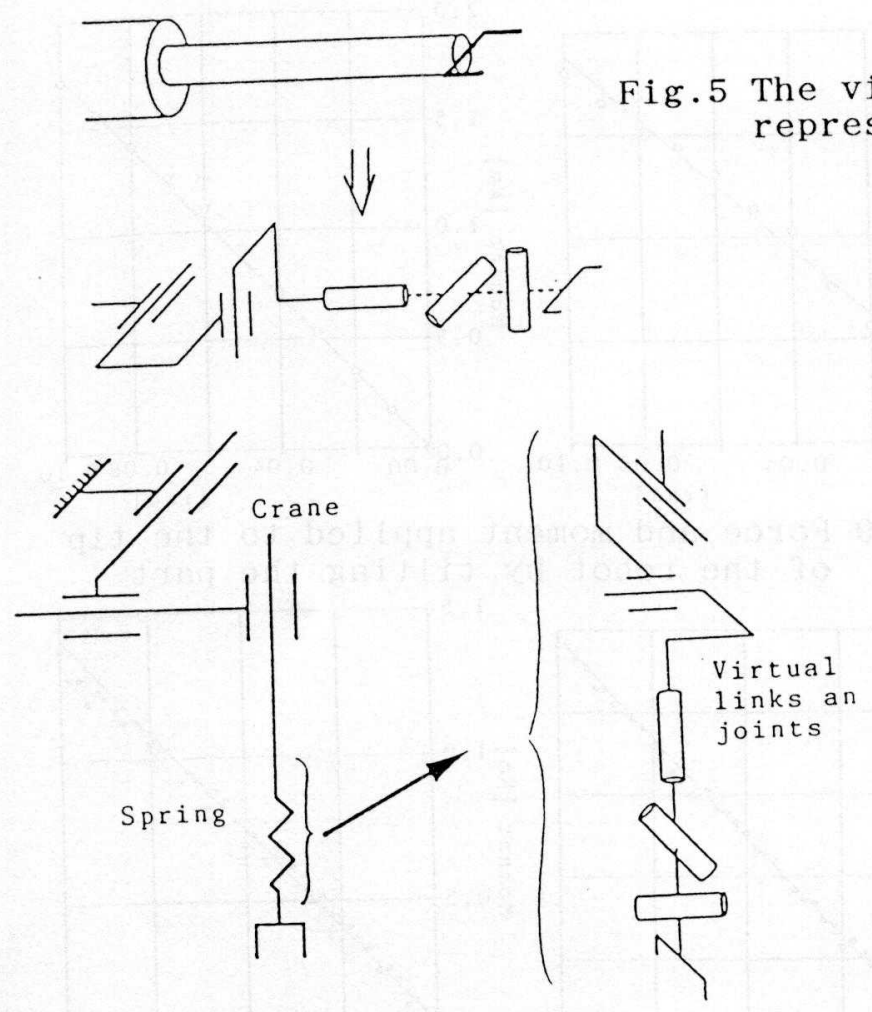

Fig. 6 Crane model with virtual links and joints

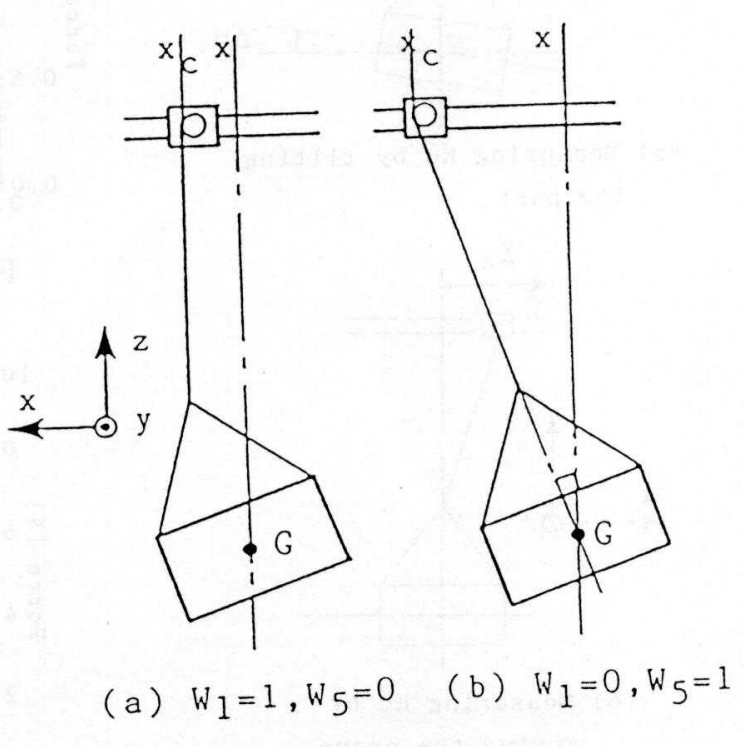

Fig. 7 Optimal solution of the crane position

\section{EXPERIMENTS OF THE COORDINATED SYSTEM}

\subsection{The Experimental System}

Fig. 1 illustrates the schematic view of the experimental system. The crane consists of 3 degrees of freedom: a $x-y$ table driven by DC motors with ball screws and a wire winding-up mechanism with worm wheel gears. It is $2.5[\mathrm{~m}]$ high (the $\mathrm{z}$ direction) and $1[\mathrm{~m}]$ wide (x) and 0.25 $[\mathrm{m}]$ deep (y) in working area. Yasukawa Motoman L-3 is a conventional industrial robot whose payload is $3 \mathrm{~kg}$. It has 5 axis force sensor at the tip of the end-effector, and a parallel beam spring is installed at the front of the force sensor. The heavy part weighs $8.9 \mathrm{~kg}$ and is fixed to the parallel beam spring. The control devises uses a personal computer Epson's PC- $386 \mathrm{~V}(20 \mathrm{MHz}$, $80386+80387)$ and 12 bit AD converters.

Let us discuss two kinds of experiments: (1) to determine the stiffness matrix and (2) to verify the algorithm of the inverse kinematics.

\subsection{Experiment to Measure the Stiffness Matrix}

The following two experiments are for the verification of stiffness matrix. Using the parameters $\mathrm{L}=0.9[\mathrm{~m}]$ and $\mathrm{d}=0.25[\mathrm{~m}]$, the stiffness matrix $\mathrm{K}_{\mathrm{C}}$ is obtained theoretically as

$\mathrm{K}_{\mathrm{c}}=\left[\begin{array}{cccccc}8.7 \times 10 & 0 & 0 & 0 & 1.9 \times 10 & 0 \\ 0 & 8.7 \times 10 & 0 & -1.9 \times 10 & 0 & 0 \\ 0 & 0 & 2.7 \times 10^{5} & 0 & 0 & 0 \\ 0 & -1.9 \times 10 & 0 & 2.3 \times 10 & 0 & 0 \\ 1.9 \times 10 & 0 & 0 & 0 & 2.3 \times 10 & 0 \\ 0 & 0 & 0 & 0 & 0 & 1.2 \times 10^{-4}\end{array}\right]$




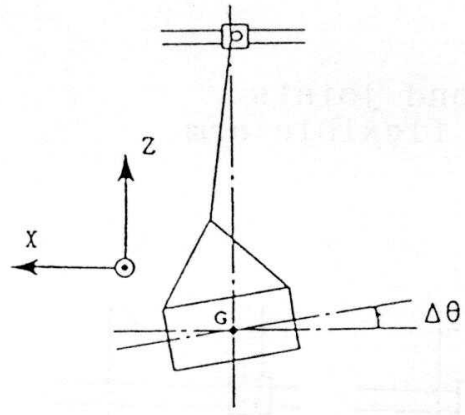

(a) Measuring Kc by tilting the part

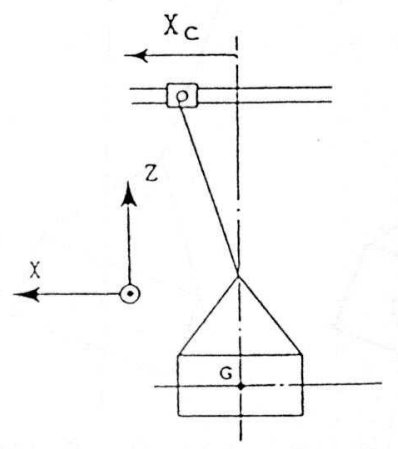

(b) Measuring Kc by moving the crane

Fig. 8 Verification of the stiffness matrix of the crane
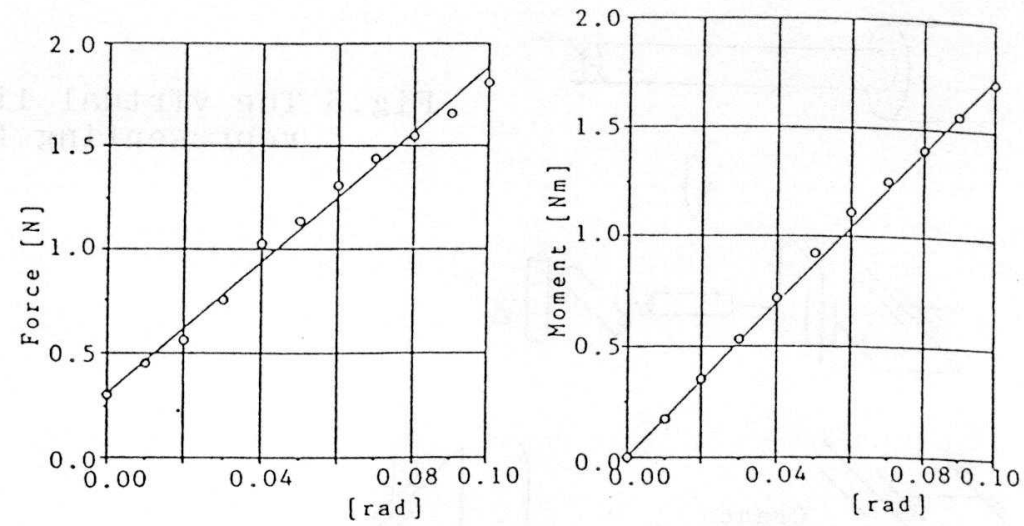

Fig.9 Force and moment applied to the tip of the robot by tilting the part
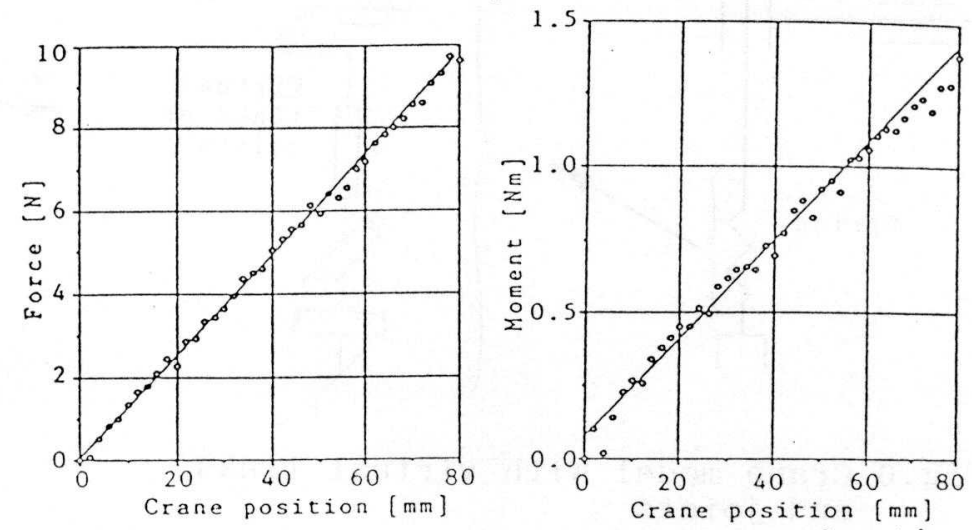

Fig. 10 Force and moment applied to the tip of the robot by moving the crane

(1) Measuring $\mathrm{K}_{\mathrm{C}}$ by tilting the part: The suspended part is positioned just underneath of the crane and its posture is set horizontally as shown in Fig.8(a). While the wrist of the robot is rotating from 0 to $0.1[\mathrm{rad}]$, the force along the $\mathrm{x}$ axis, $\mathrm{f}_{\mathrm{X}}$, and the moment about the $\mathrm{y}$ axis, $\mathrm{m}_{\mathrm{y}}$, of the robot coordinate system are measured. Fig. 9 indicates the results and then 2 components of $\mathrm{K}_{\mathrm{C}}, \mathrm{K}_{15}=1.6 \times 10$ and $\mathrm{K}_{55}=1.7 \times 10$, are obtained. These values deviate in $16 \%$ and $22 \%$ from the computed value, $1.9 \times 10$ and $3.2 \times 10$ respectively. But the errors can be explained by the kinematical errors of wire tension system and the bad estimation of the wire length.

(2) Measuring $\mathrm{K}_{\mathrm{C}}$ by moving the crane: The part and the robot are positioned stably in keeping the part horizontally as shown in Fig.8(b). While the crane is moving in the $x$ direction, $f_{x}$ and $m_{y}$ are measured. Fig. 10 indicates the results and then 3 components of $\mathrm{K}_{\mathrm{C}}, \mathrm{K}_{11}=1.2 \times 10^{2}, \mathrm{~K}_{51}=1.6 \times 10$ and $K_{15}=1.5 \times 10$, are obtained. Equivalent components $K_{15}$ and $K_{51}$ show similar to each other but deviate in $16 \%$ from the computed value. The reason can be explained in the same way as above.

Even though the measured components of $\mathrm{K}_{\mathrm{C}}$ contain relatively high errors against the computed value, we conclude that the definition of the stiffness matrix is proper. It is because the relationships among the components are reasonable. Therefore, the stiffness matrix obtained in these experiments will be used in the following algorithm.

\subsection{Experiment to Verify the Algorithm of the Inverse Kinematics}

In order to verify the algorithm of the inverse kinematics, the position of the crane is obtained where either $f_{\mathrm{X}}$ or $\mathrm{m}_{\mathrm{y}}$ becomes minimum respectively.

The part is tilted in $0.05[\mathrm{rad}]$ about the $y$ axis of the robot coordinate system and the robot is stably positioned as shown in Fig. 11 . While the crane is moving in $0.080[\mathrm{~m}]$ along the $x$ axis, $f_{X}$ and $m_{y}$ are measured. The result is shown in Fig.12. The optimal positions of the crane for the abovementioned conditions are obtained in the experiment as 


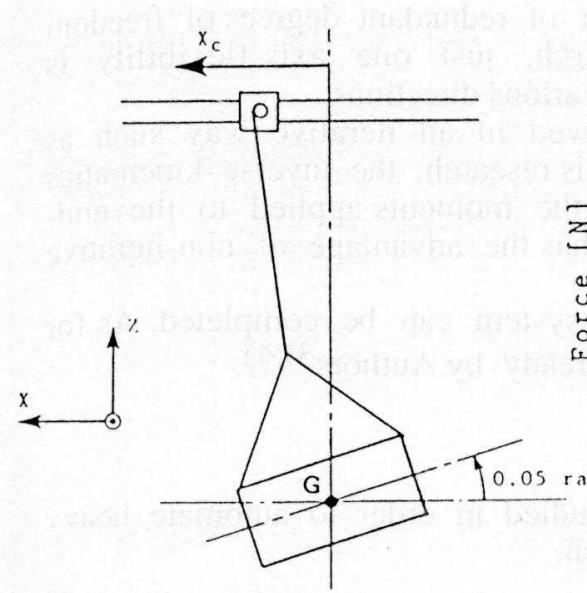

Fig.11 Verification of the algorithm of the inverse kinematics
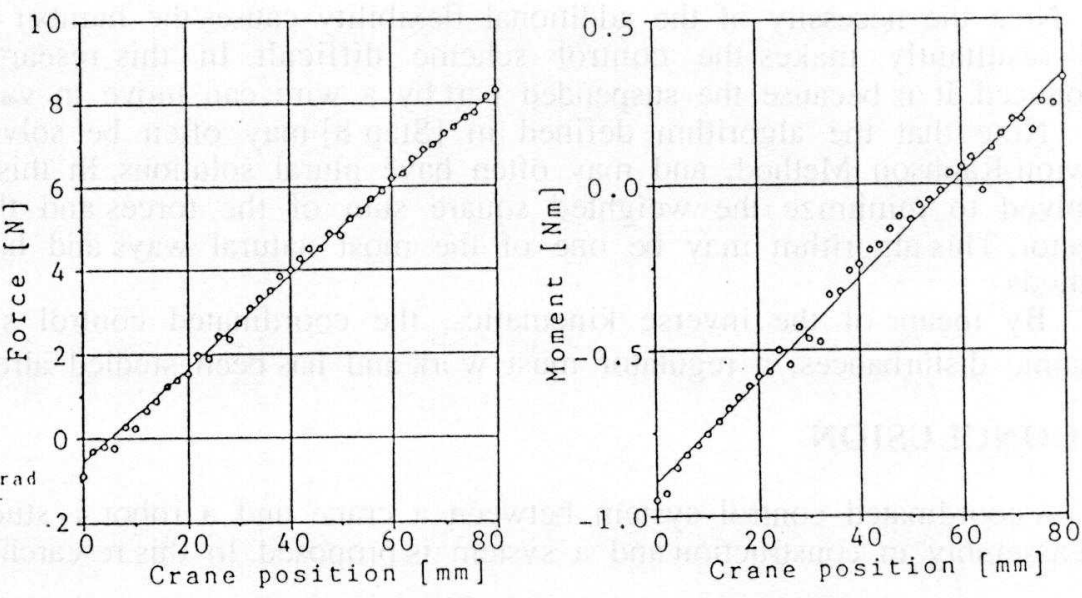

Fig. 12 Force and moment applied to the tip of the robot by moving the crane

$f_{X}=0: x_{C}=6.2 \times 10^{-3}[\mathrm{~m}]$

$\mathrm{m}_{\mathrm{y}}=0: \mathrm{x}_{\mathrm{c}}=5.8 \times 10^{-2}[\mathrm{~m}]$

Using the stiffness matrix obtained in 4.2 , the optimal positions are computed as

$f_{X}=0: x_{C}=6.7 \times 10^{-3}[\mathrm{~m}]$

$\mathrm{m}_{\mathrm{y}}=0: \mathrm{x}_{\mathrm{c}}=5.3 \times 10^{-2}[\mathrm{~m}]$

These values coincide practically to each other correspondingly and thus we conclude that the algorithm is verified.

\section{PROCEDURE TO DEFINE A COORDINATED CONTROL SYSTEM}

In order to use a conventional industrial robot in coordinated control with a crane, the kinematics and the statics of the coordinated control have been studied in this paper. In wire suspension system using the gravity, the settling pose of a suspended part can be determined by both the geometrical relationships and the static equilibrium. A kinematic loop of the coordinated two mechanisms produces another geometrical constraint. However, the system may have the redundancy in kinematics, when the system includes flexibility in structure. Thus, it is important to solve the inverse kinematics.

Here, the sequence of the design of the coordinated system is summarized:

[Step 1] Obtain the stiffness matrix of the suspended mass, $K_{C}$, by applying Eq.(2).

[Step 2] Measure experimentally the stiffness matrix, $\mathrm{K}_{\mathrm{C}}$, and correct $\mathrm{K}_{\mathrm{C}}$.

[Step 3] Determine the positioning error $d \mathbf{x}$ and the allowable force on the robot side $\mathbf{F}_{\mathrm{r}}$, according to the strength of the robot.

[Step 4] Measure the stiffness matrix of the robot at the tip of the end-effector, $\mathrm{K}_{\mathbf{r}}$.

[Step 5] Calculate Eq.(1). If the inequality is satisfied, go to Step 6. If not, adjust $\mathrm{K}_{\mathrm{r}}$ by installing additional flexibility and go to [Step 4].

[Step 6] Make a model of virtual links and joints. Determine the redundant degrees of freedom by Eq.(5).

[Step 7] Define the restriction algorithms to fix the redundancy in kinematics.

[Step 8] Complete an algorithm of the inverse kinematics. 
Note the necessity of the additional flexibility causes the number of redundant degrees of freedom and resultantly makes the control scheme difficult. In this research, just one axis flexibility is introduced. It is because the suspended part by a wire can move in various directions.

Note that the algorithm defined in [Step 8] may often be solved in an iterative way such as Newton-Raphson Method, and may often have plural solutions. In this research, the inverse kinematics is solved to minimize the weighted square sum of the forces and the moments applied to the endeffector. This algorithm may be one of the most natural ways and has the advantage of non-iterative methods.

By means of the inverse kinematics, the coordinated control system can be completed. As for dynamic disturbances, a regulator must work and has been studied already by Authors 3$), 4$ ).

\section{CONCLUSION}

A coordinated control system between a crane and a robot is studied in order to automate heavy part assembly in construction and a system is proposed. In this research,

(1) Design methodology of compliance is studied.

(2) Mechanisms with flexibility are analyzed; it is clarified that the proposed coordinated mechanism has redundancy in kinematics.

(3) Stiffness matrix is defined theoretically with a model and is determined in experiment.

(4) To achieve position control of both the crane and the robot, the inverse kinematics is proposed, where stiffness matrix of the crane is necessary. The algorithm is verified in experiment.

The proposed assembly system has the following characteristics:

- for heavy parts assembly

- coordinated control system between a crane and an industrial robot

- positioning control

- simple compliance installed on the robot to accommodate the mutual positioning error

\section{REFERENCE}

1) Matsui,T., et al., 1982, Application of Robots to Assembly Lines, THE HITACHI HYORON, Vol.64, No.12, 33-38(in Japanese).

2) Patarinski,S.P., et al.,1985, Robot-Balancing Manipulator Cooperation for Handling of Heavy Parts, Proc. of 15th Int. Symp. Industrial Robots, 649-656.

3) Arai,T., Osumi,H., Ohta,J.,1988, Crane Control System with an Industrial Robot, Proc. 5th Int. Symp. on Robotics in Constr., Vol.2, 747-754.

4) Arai,T., Ohsumi,H.,1990, Assembly of Suspended Heavy Works, Annals of the CIRP, 39,37-40.

5) Yoshikawa,T., 1985, Manipulability and Redundancy Control of Robotic mechanisms, Proc. of 1985 IEEE Int. Conf. on Robotics and Automation, 1004-1009. 